\title{
Changing patterns of T lymphocyte subsets after kidney transplantation according to induction immunosuppressant: single center prospective observational study
}

\author{
Hyung Duk Kim ${ }^{1}$, Hyunjoo Bae ${ }^{2}$, Sang Hun Eum ${ }^{1}$, Hanbi Lee ${ }^{1}$, Eun Jeong Ko ${ }^{1}$, Chul Woo Yang ${ }^{1}$, Eun-Jee $\mathrm{Oh}^{3}$, \\ Byung Ha Chung ${ }^{1}$

\footnotetext{
${ }^{1}$ Department of Internal Medicine-Nephrology, The Catholic University of Korea, Seoul St. Mary's Hospital, Seoul, Korea

${ }^{2}$ Department of Biomedical Science, Graduate School, The Catholic University of Korea, Seoul, Korea

${ }^{3}$ Department of Laboratory Medicine, The Catholic University of Korea, Seoul St. Mary's Hospital, Seoul, Korea
}

Background: The aim of this study is to investigate the changing patterns of $T$ cell subsets during early post-transplant period according to the type of induction therapy (anti-thymocyte globulin [ATG] vs. basiliximab).

Methods: We conducted prospective observational study for 157 patients who underwent kidney transplantation (KT) in Seoul St. Mary's Hospital from May 2018 to November 2020. A baseline blood sample was collected within 5 days before the kidney transplant, and additional blood samples were collected and analyzed at 4 and 12 weeks after the kidney transplant. We compared the change of each T cell subsets between patients who took ATG $(n=62)$ and basiliximab $(n=95)$ using flow cytometric study of peripheral blood mononuclear cells.

Results: At baseline, all of the CD4+ and CD8+ T cell subsets did not show significant differences. However, changing pattern of T cell subsets showed significant difference according to the type of induction therapy at 4 weeks and 12 weeks after KT. In the ATG group, a significant decrease in CD4+ T cells was observed from week 4 and continued until week 12. CD8+ T cells showed no change until week 4 , but increased at week 12. A significant increase in the CD4+CD161+ and CD8+CCR7-CD45RA+ T cell subsets was observed at 4 and 12 weeks in the ATG group, and CD8+CCR7+ T cell subset decreased. CD8+CD28nullCD57+ T cell was decreased at 4 weeks and recovered to baseline levels at 12 weeks. In basiliximab group, CD8+CCR7+ T cell expression was decreased and CD8+CCR7-CD45RA+ T cell expression was increased at 12 weeks compared to baseline.

Conclusions: In this study, we observed CD4+CD161+ and CD8+CCR7-CD45RA+ T cells activation in patients with ATG induction in comparison with basiliximab. The correlation between T cell subset changes and clinical outcome could not be confirmed in our study.

Corresponding author: Byung Ha Chung

E-mail: chungbh@catholic.ac.kr

(c) The Korean Society for Transplantation

This is an Open Access article distributed under the terms of the Creative Commons Attribution Non-Commercial License (http://creativecommons.org/licenses/by-nc/4.0/) which permits unrestricted non-commercial use, distribution, and reproduction in any medium, provided the original work is properly cited. 